\title{
Solución numérica de procesos de transferencia de calor en aleaciones con condiciones de contorno no lineales
}

\author{
F. Alhama*, D. Alcaraz** y C.F. González-Fernández*
}

\begin{abstract}
Resumen Las propiedades térmicas de numerosas aleaciones, especialmente la capacidad calorífica, acusan una marcada dependencia con la temperatura tanto en los cambios de fase como en las transformaciones alotrópicas. Este hecho, junto con la aplicación de condiciones de contorno de convección, radiación o ambas, simultáneamente, aumentan la complejidad del problema de transferencia de calor en los procesos transitorios debido a la no linealidad inherente. El modelo numérico propuesto para la solución de este problema es capaz de asumir las hipótesis anteriores así como las posibles dependencias de la conductividad térmica con la temperatura. Se presentan aplicaciones a procesos térmicos en aleaciones.
\end{abstract}

Palabras clave Conducción térmica. Aleaciones. Método redes.

\section{Numerical solution for heat transfer processes in alloys with non lineal boundary conditions}

\begin{abstract}
Thermal properties of a great number of alloys, especially specific heat, show a sharply temperature dependence both during the phase change and the allotropic transformations. This fact, in conjunction with the existence of convection or radiation boundary conditions, or both simultaneously, increase the complexity of the transient heat transfer problem because of the inherent no linearity. The numerical model proposed for the solution of this problem is able to assume the above hypothesis as well as the temperature dependencies of the thermal conductivity. Applications to alloys are presented.
\end{abstract}

Keywords Heat conduction. Alloys. Network method.

\section{INTRODUCCIÓN}

Los procesos transitorios de transferencia de calor en medios sólidos, regidos por la ecuación de conducción ${ }^{[1 ~ y ~ 2], ~ e s t a ́ n ~ c o n t r o l a d o s ~ p o r ~ l a s ~ c a r a c t e r i ́ s t i-~}$ cas térmicas conductividad, $\mathrm{k}, \mathrm{y}$ calor específico, $\mathrm{c}_{\mathrm{e}}$. Estas propiedades son, en general, dependientes de la temperatura, particularmente en los procesos de cambio de fase o de transformaciones alotrópicas, lo que convierte la conducción en un proceso no lineal cuya solución requiere técnicas numéricas tales como diferencias finitas, elementos finitos, método entálpico, etc. ${ }^{[1 \text { y } 3]}$. Si a esto se añade la existencia de condiciones de contorno, a su vez, no lineales, tales como convección natural, radiación o convección más radiación, la complejidad del problema crece aún más. En la literatura científica se encuentra información (tabulada o gráfi- ca) de las dependencias $k(T), c_{e}(T)$ o de la difusividad térmica $\alpha(T), \alpha=k /\left(\rho c_{e}\right)$ con $\rho$ la densidad del sólido ${ }^{[4}$ y 5$]$.

El objetivo del presente trabajo es aplicar un modelo sencillo basado en el método de simulación por redes (MSR) ${ }^{[6]}$, para obtener soluciones numéricas precisas y rápidas de procesos de transferencia térmica en aleaciones, incluyendo procesos de cambio de fase, bajo condiciones de contorno arbitrarias (no lineales). La solución de este problema proporciona información fundamental para el uso de los diagramas RPTT ${ }^{[7]}$ y la determinación de propiedades mecánicas de estas aleaciones derivadas de los diferentes tipos de tratamiento térmico ${ }^{[8]}$. La aplicación del método se realiza en dos etapas: (i) diseño de un modelo en red a partir de las ecuaciones del modelo matemático y (ii) simulación en un software de resolución de circuitos tal como PSPICE ${ }^{[9]}$.

(*) Universidad Politécnica de Cartagena. Dpto. Física Aplicada. Campus Muralla del Mar. 30202 Cartagena (Murcia, España).

(**) Universidad Politécnica de Cartagena. Dpto. de Ing. de Materiales y Fabricación. Campus Paseo Alfonso XIII. 30203 Cartagena (Murcia, España). 
A pesar de la complejidad del problema, gracias a la versatilidad de ciertos componentes de las librerías de este software, que permiten implementar directamente cada uno de los miembros de la ecuación en diferencias finitas, y de las condiciones de contorno, sólo son necesarios unos pocos componentes para implementar el modelo. Además, el investigador no necesita efectuar manipulaciones matemáticas de las ecuaciones en diferencias finitas de las que se deriva el modelo, ya que es el software quien se encarga de este trabajo, ni precisa resolver los problemas de convergencia de la solución (tarea que se deja también al software PSPICE). Se presentan aplicaciones a aleaciones que permiten obtener los campos térmicos y flujos de calor en problemas 1-D con y sin cambio de fase.

\section{NOMENCLATURA}

$\mathrm{c}_{\mathrm{e}} \quad$ calor específico $\left(\mathrm{J} \mathrm{kg}^{-1} \mathrm{C}^{-1}\right)$

h coeficiente de convección $\left(\mathrm{Wm}^{-2} \mathrm{C}^{-1}\right)$

$\mathrm{f}$ función continua arbitraria

j flujo de calor $\left(\mathrm{Wm}^{-2}\right)$

$\mathrm{k}$ conductividad térmica $\left(\mathrm{Wm}^{-1} \mathrm{C}^{-1}\right)$

$\mathrm{r}$ posición $(\mathrm{m})$

$\mathrm{t}$ tiempo (s)

$\mathrm{T}$ temperatura $(\mathrm{K})$

$\mathrm{T}_{\text {ini }}$ Temperatura inicial $(K)$

$\begin{array}{ll} & \text { Símbolos griegos: } \\ \varepsilon & \text { emisividad superficial } \\ \rho & \text { densidad }\left(\mathrm{kg} \mathrm{m}^{-3}\right) \\ \sigma & \text { constante de Stefan Boltzmann }\left(\mathrm{Wm}^{-2} \mathrm{~K}^{-4}\right)\end{array}$

\section{ENUNCIADO DEL PROBLEMA}

Un sólido, 1-D, cuya temperatura inicial es $T_{\text {ini }}$, se somete a procesos de calentamiento y/o enfriamiento bajo la acción de un cambio en sus condiciones de contorno. La conductividad y calor específico son dependientes de la temperatura y pueden expresarse mediante funciones matemáticas continuas de cualquier tipo. Las condiciones de contorno pueden ser arbitrarias ${ }^{[2]}$, incluyendo convección libre o forzada y condición de radiación o ambas simultáneamente, como es frecuente en los procesos de tratamiento térmico.

Partiendo de la ecuación de Fourier, $j=-k \nabla$ (T) que relaciona la densidad de flujo de calor, $\mathrm{j}$, con el gradiente térmico, $\nabla(\mathrm{T})$, la aplicación del balance térmico a un elemento de volumen de un medio homogéneo e isótropo, sin fuentes ni sumideros de calor, conduce a la ecuación:

$$
\nabla[\mathrm{k} \nabla(\mathrm{T})]=\rho \mathrm{c}_{\mathrm{e}}(\partial \mathrm{T} / \partial \mathrm{t})
$$

Para $\mathrm{k}$ y $\mathrm{c}_{\mathrm{e}}$ pueden asumirse dependencias arbitrarias, expresadas por funciones continuas o funciones lineales, a tramos que permiten elaborar una buena aproximación de cualquier tipo de dependencia pueden ser también asumidas para $\mathrm{k} \mathrm{y} \mathrm{c}_{\mathrm{e}}$.

$$
\begin{aligned}
& k(T)=f_{k}(T) \\
& c_{e}(T)=f_{c}(T)
\end{aligned}
$$

Por otro lado, las ecuaciones asociadas con las condiciones de contorno e inicial son:

$$
\begin{gathered}
\mathrm{T}(\mathrm{t})=\mathrm{T}_{\mathrm{s}}(\mathrm{r}, \mathrm{t}) \\
-\mathrm{k}(\partial \mathrm{T} / \partial \mathrm{n})_{\mathrm{s}}=\mathrm{j}(\mathrm{r}, \mathrm{t}) \\
\mathrm{h}\left(\mathrm{T}-\mathrm{T}_{\infty, \mathrm{con}}\right)_{\mathrm{s}}=-\mathrm{k} \partial \mathrm{T} / \partial \mathrm{n} \\
\sigma \varepsilon\left(\mathrm{T}^{4}-\mathrm{T}_{\infty, \mathrm{rad}}^{4}\right)_{\mathrm{s}}=-\mathrm{k} \partial \mathrm{T} / \partial \mathrm{n} \\
\mathrm{h}\left(\mathrm{T}-\mathrm{T}_{\infty, \mathrm{con}}\right)_{\mathrm{s}}+\sigma \varepsilon\left(\mathrm{T}^{4}-\mathrm{T}_{\infty, \mathrm{rad}}{ }^{4}\right)_{\mathrm{s}}=-\mathrm{k} \partial \mathrm{T} / \partial \mathrm{n} \\
\mathrm{T}_{(\mathrm{t}=0)}=\mathrm{T}_{\text {ini }}(\mathrm{r})
\end{gathered}
$$

Las ecuaciones (4a) y (4b) representan, respectivamente, las condiciones isoterma y de flujo incidente constante en la superficie del material (condiciones incompatibles entre sí y con cualquier otra condición térmica). A su vez, las ecuaciones (4c) y (4d) representan, respectivamente, condiciones convectiva y de radiación. La simultaneidad de ambas condiciones viene expresada por la ecuación (4e). Por último, la ecuación (5) representa la condición inicial.

En estas ecuaciones: $\partial \mathrm{T} / \partial \mathrm{n}$ es el gradiente térmico normal en la superficie; $T_{s}$, la temperatura en la superficie; $\mathrm{T}_{\infty, \mathrm{con}} \mathrm{y} \mathrm{T}_{\infty, \text { rad }}$, las temperaturas de referencia para la convección y radiación (habitualmente, $T_{\infty, \text { con }}=T_{\infty, \text { rad }}$ ), respectivamente; $h$ es el coeficiente de convección (función de $\mathrm{T}_{\mathrm{s}}-\mathrm{T}_{\infty \text {,con }}$ en convección natural); $\sigma$, la constante de Stefan Boltzmann; $\varepsilon$, la emisividad de la superficie; y $r$, la coordenada espacial. Las correlaciones entre $h$ (o su expresión adimensional el número de Nusselt) y el resto de las magnitudes térmicas y geométricas del problema son de la forma $N_{u}=\left(h l_{o}\right) / k=$ $f\left(G_{r}, P_{r}, R_{a}\right){ }^{[2]}$, donde $G_{r}, P_{r}$ y $R_{a}$ son los números adimensionales de Grashof, Prandtl y Rayleigh, respectivamente, y $l_{o}$ es una longitud característica del problema. Estas correlaciones tiene nombres específicos según la geometría.

\section{MODELO NUMÉRICO}

El modelo en red, derivado de la aplicación del MSR ${ }^{[6]}$, se muestra en la figura 1. Para mayor 
Solución numérica de procesos de transferencia de calor en aleaciones con condiciones de contorno no lineales F. AlHAMA, D. AlCARAZ y C.F. GONZÁlez-FerNáNDEZ

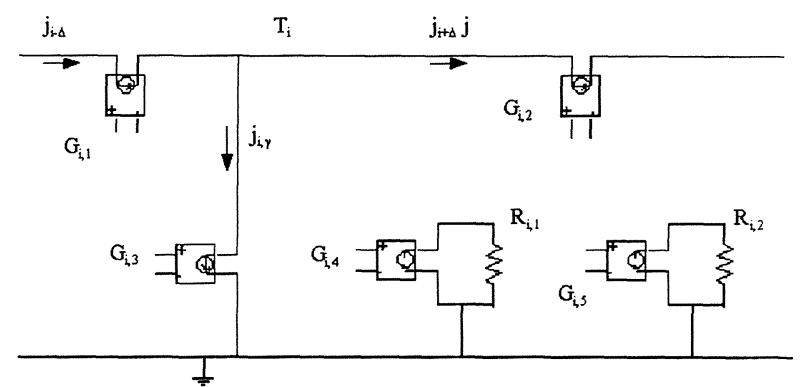

Figura 1. Modelo en red de un elemento de volumen.

Figure 1. Network model for a volume element.

claridad, en la figura 2 se muestra la nomenclatura empleada.

Las corrientes de los generadores $\mathrm{G}_{\mathrm{i}, 1}, \mathrm{G}_{\mathrm{i}, 2}$ y $\mathrm{G}_{\mathrm{i}, 3}$ son $\mathrm{j}_{\mathrm{i}-\Delta}=\mathrm{S}_{\mathrm{i}-\Delta} k_{\mathrm{i}}\left(\mathrm{T}_{\mathrm{i}-\Delta}-\mathrm{T}_{\mathrm{i}}\right) /\left(\Delta \mathrm{r}_{\mathrm{i}} / 2\right), \mathrm{j}_{\mathrm{i}+\Delta}=\mathrm{S}_{\mathrm{i}+\Delta}$ $k_{\mathrm{i}}\left(\mathrm{T}_{\mathrm{i}}-\mathrm{T}_{\mathrm{i}+\Delta}\right) /\left(\Delta \mathrm{r}_{\mathrm{i}} / 2\right)$ y $\mathrm{j}_{\mathrm{i}, \mathrm{c}}=\Gamma_{\mathrm{i}} \rho \mathrm{c}_{\mathrm{e}, \mathrm{i}}\left(\mathrm{dT} \mathrm{T}_{\mathrm{i}} / \mathrm{dt}\right) ;$ así, $\mathrm{j}_{\mathrm{i}-\Delta}-$ $j_{i+\Delta}-j_{i, c}=0$, ecuación en diferencias finitas resultado de aplicar la reticulación espacial a la ecuación (1). Para coordenadas planas $S_{i-\Delta}=S_{i+\Delta}$ y $\Gamma_{i}=$ $S_{i} \Delta r_{i}$, lo que permite simplificar las expresiones anteriores. Los generadores de tensión $G_{i, 4}$ y $G_{i, 5}$ proporcionan a su salida los valores de conductividad y calor específico en el centro de la celda, $k_{i}=$ $f_{k}\left(T_{i}\right)$ y $c_{e, i}=f_{c}\left(T_{i}\right) . R_{i, 1}$ y $R_{i, 2}$ son resistencias requeridas por el software. La interconexión de los elementos de volumen y la inclusión de las condiciones de contorno se lleva a cabo de manera inmediata, siguiendo unas pocas reglas de programación muy intuitivas ${ }^{[9]}$. La figura 3 muestra los elementos necesarios para implementar las condiciones de convección y/o radiación $(r=0)$, que usa una fuente del tipo $\mathrm{G}$, y la condición adiabática $(\mathrm{r}=\mathrm{L}$, espesor de la pieza) que usa una resistencia de valor infinito".

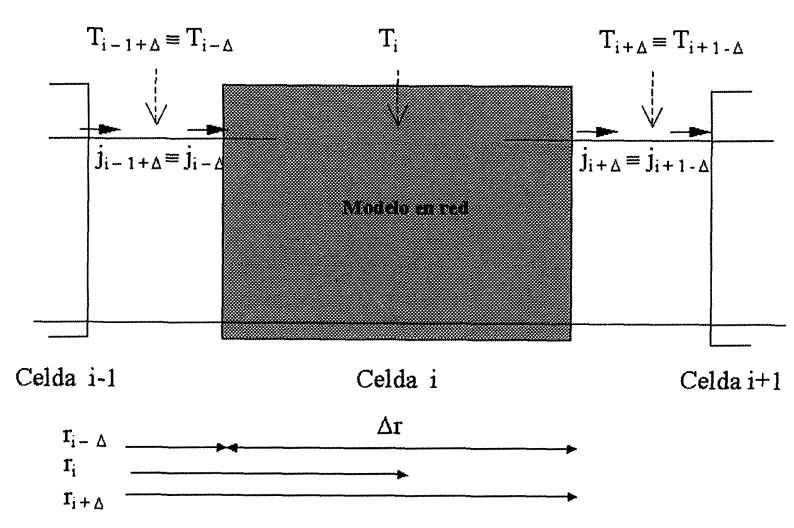

Figura 2. Nomenclatura del elemento de volumen o celda.

Figure 2. Nomenclature of volume element or cell.

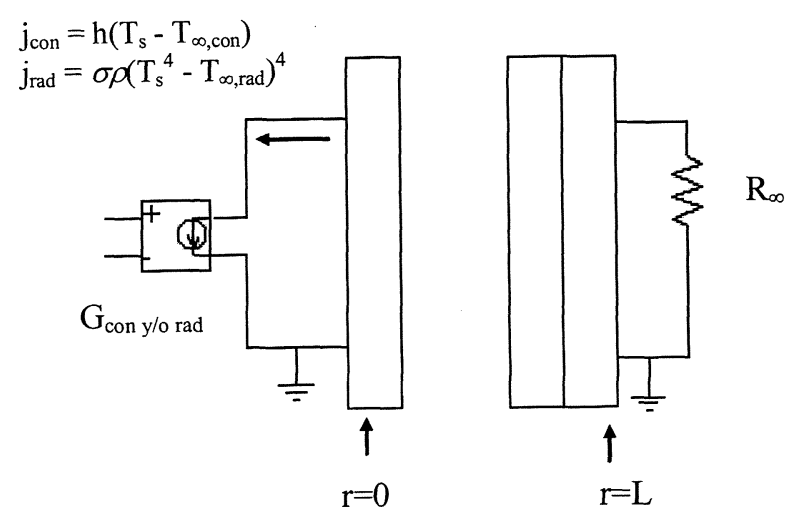

Figura 3. Modelo de condiciones de contorno.

Figure 3. Boundary conditions model.

\section{APLICACIÓN}

Los materiales seleccionados son la aleación de aluminio A201 y una fundición de hierro con grafito compacto (CGI). Las dependencias $k(T)$ y $c_{e}(T)$ para calentamiento y enfriamiento se muestran en las figuras 4 y $5^{[4]}$.

La Figura 6a) muestra la temperatura en la superficie de convección (o radiación, curva III) de la aleación A201 durante un proceso de enfriamiento. La otra superficie del medio se somete a condición adiabática. Los flujos de calor en la superficie convectiva, o de radiación, se muestran en la Figura 6b). Los valores numéricos son: $\mathrm{L}=0,05 \mathrm{~m}$, $\mathrm{T}_{\mathrm{ini}}=1.000 \mathrm{~K}, \mathrm{~T}_{\infty, \mathrm{con}}=\mathrm{T}_{\infty, \mathrm{rad}}=700 \mathrm{~K}$. El medio se ha dividido en 100 elementos de volumen. Las figuras 7a) y $7 \mathrm{~b}$ ) representan las mismas magnitudes para procesos de calentamiento y enfriamiento de la CGI. La convección se realiza con $h=500$. Los valores numéricos son: $L=0,05 \mathrm{~m}$, $\mathrm{T}_{\text {ini }}=1.550 \mathrm{~K}, \mathrm{~T}_{\infty, \mathrm{con}}=\mathrm{T}_{\infty, \mathrm{rad}}=800 \mathrm{~K}$. En todos los casos, el medio se ha dividido en 100 elementos de volumen. Los tiempos de computación en todos los casos ejecutados no superan los $20 \mathrm{~s}$ en un ordenador personal Pentium IV.

\section{CONCLUSIONES}

Se presenta un modelo simple y eficaz, basado en el método de simulación por redes, que permite la simulación de procesos transitorios de transferencia de calor fuertemente no lineales con condiciones de contorno arbitrarias. Se aplica dicho modelo a tratamientos térmicos de aleaciones, incluyendo procesos alotrópicos y cambios de fase, obteniéndose los campos térmicos y flujos de 
calor simultáneamente, sin necesidad de manipulación matemática de las ecuaciones en derivadas parciales del modelo matemático por parte del usuario.
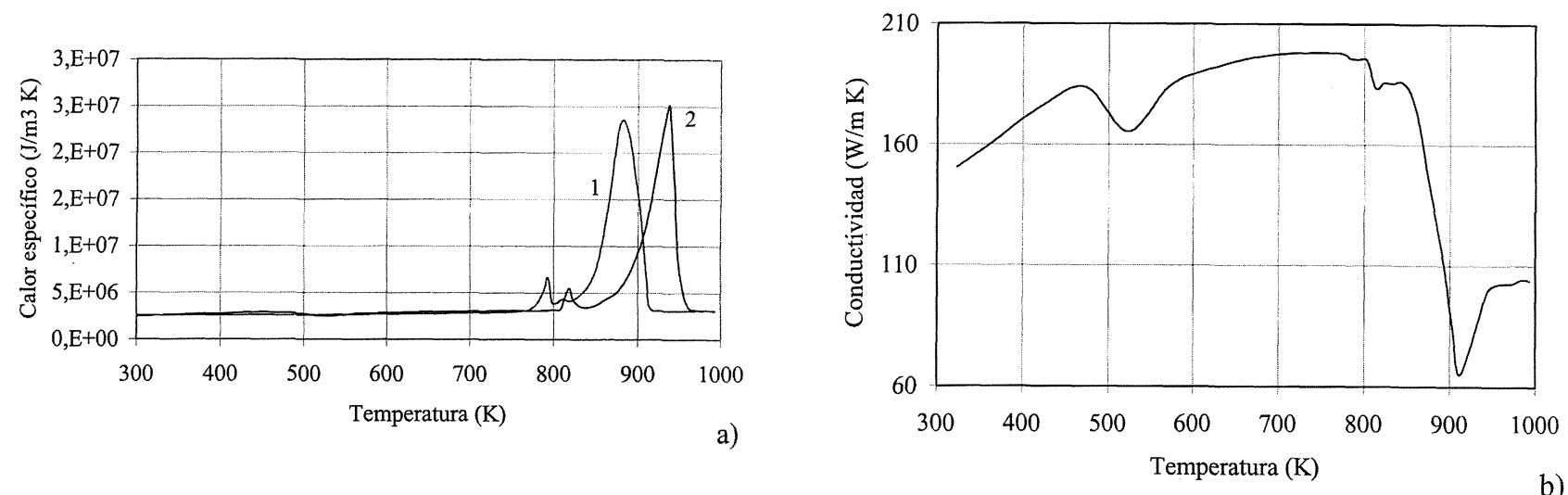

Figura 4. a) Aleación A201. $c_{e}$, 1: enfriamiento, 2: calentamiento; b) Aleación A201: conductividad térmica (k).

Figure 4. a) A201 alloy. $c_{e}$, 1: cooling, 2: heating; b) A201 alloy: thermal conductivity (k).
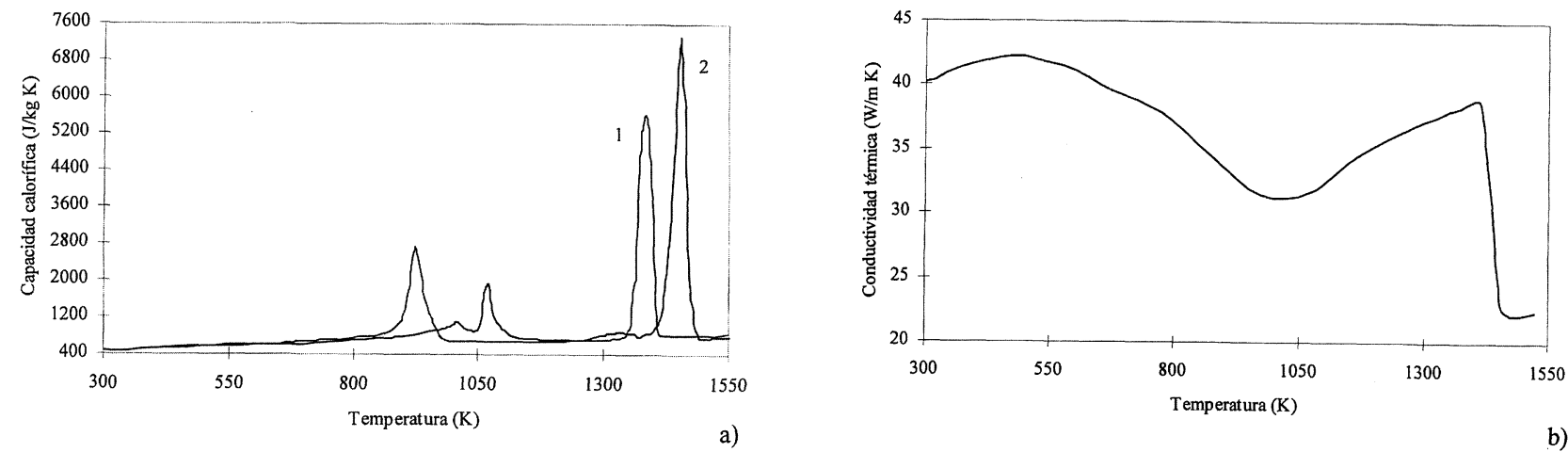

a)

Figura 5. a) CGI. $c_{e}, 1$ : enfriamiento, 2: calentamiento; b) CGI: conductividad térmica (k).

Figure 5. a) CGI. $c_{e}$, 1: cooling, 2: heating; b) CGI: thermal conductivity (k).
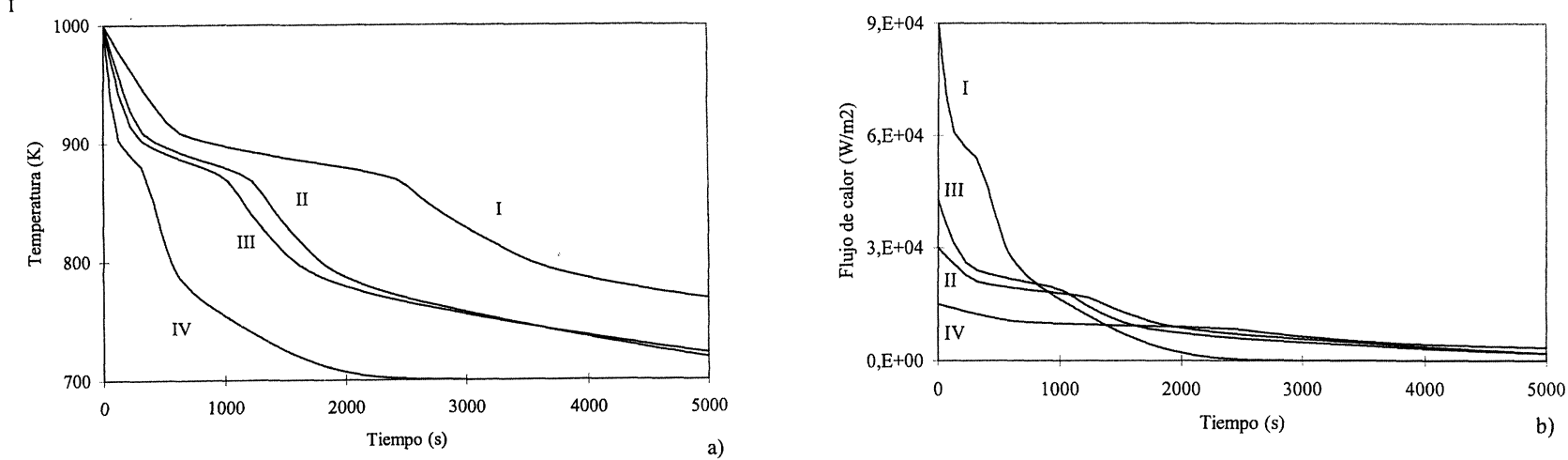

Figura 6. a) I: $h=50, I I: h=100, I V: h=300$; III: enfriamiento por radiación, $h=0 ; b)$ I: $h=50$, II: $h=100, I V: h=300$; III: enfriamiento por radiación, $h=0$.

Figure 6. a) I: $h=50$, II: $h=100, I V: h=300$; III: radiative cooling, $h=0 ; b) ~ I: h=50, I 1: h=100, I V: h=300 ;$ III: radiative cooling, $h=0$. 
Solución numérica de procesos de transferencia de calor en aleaciones con condiciones de contorno no lineales

F. AlHAMA, D. AlCARAZ y C.F. GONZÁLEZ-FERNÁNDEZ
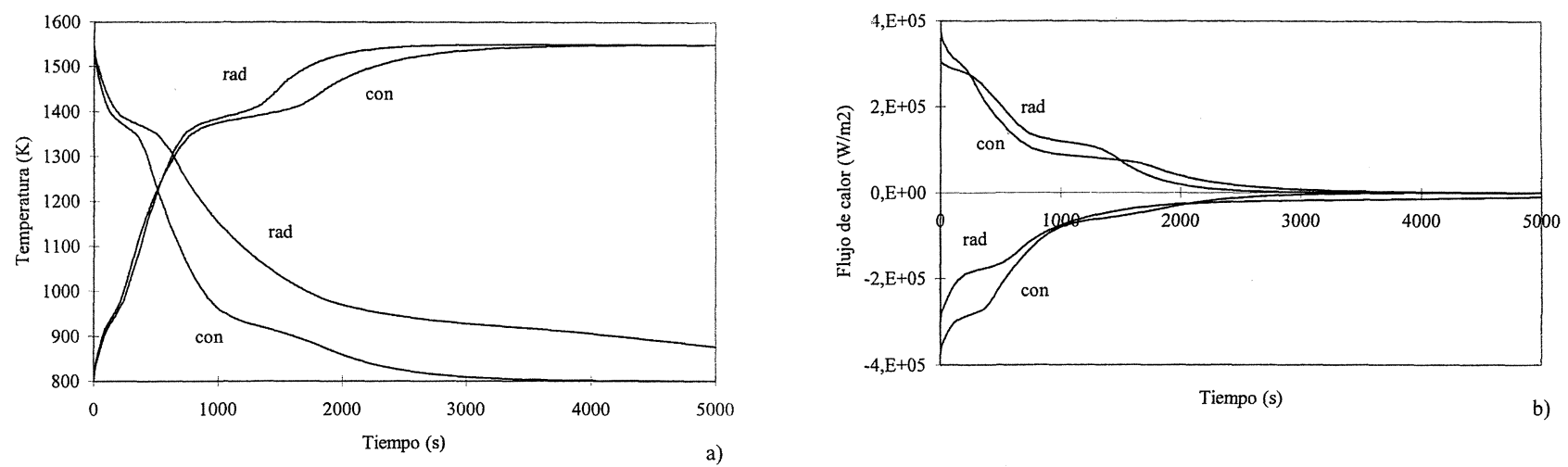

Figura 7. a) Curvas de enfriamiento y calentamiento de la $C G I$; b) Curvas de enfriamiento y calentamiento de la CGI.

Figure 7. a) Cooling and heating curves for $C G 1$; b) Cooling and heating curves for CGI.

\section{REFERENCIAS}

[1] H.S. Carslaw y J.C. Jaeger, Conduction of heat in solids, Oxford Univ. Press, Oxford, Reino Unido, 1959.

[2] F. Kreith y M.S. Born, Principles of heat transfer, PWS Publishing Company, Londres, Reino Unido, 1997.

[3] A. Salazar y A. Campo, Rev. Metal. Madrid 29 (1993) 292-299.

[4] ASM, ASM Handbook, Vols. I and II., Ohio, USA, 2002.

[5] AUBURN UNIVERSITY'S SOLIDIFICATION DESIGN CENTER, Alabama, USA, 2001, http://metalcasting.auburn.edu.
[6] C.F. GonzÁlez-FernándeZ y F. Alhama, Heat Transfer and the Network Simulation Method. In Network Simulation Method, Ed. Research Signpost, Trivamdrum, India, 2002, pp. 35-58.

[7] A.B. Quispe y S.F. Medina, Rev. Metal. Madrid 33 (1997) 382-386.

[8] I. FonseCA, J.A. Benito, I. Mejía, J. Jorba y A. Roca. Rev. Metal. Madrid 38 (2002) 249-255.

[9] MICROSIM CORPORATION, PSPICE 6.0., Microsim Corporation, Irvine, California, USA, 1994. 